\title{
A LAND COVER CHANGE DETECTION METHOD BASED ON CHANGE DIFFERENCE MAP FUSION
}

\author{
Huaqiao Xing ${ }^{1,}$, Dongyang Hou ${ }^{2}$, Miao Lu ${ }^{3}$, Jiage Chen ${ }^{1}$ \\ ${ }^{1}$ School of Surveying and Geo-informatics, Shandong Jianzhu University, Jinan, China - xinghuaqiao@126.com, \\ 1258153192@qq.com \\ ${ }^{2}$ School of Geosciences and Info Physics, Central South University, Changsha, China - houdongyang1986@163.com \\ ${ }^{3}$ Key Laboratory of Agri-informatics, Ministry of Agriculture/Institute of Agricultural Resources and Regional Planning, Chinese \\ Academy of Agricultural Sciences, Beijing, China - 283857488@qq.com
}

KEY WORDS: Change Detection, Remote Sensing Image, Change Difference Map Fusion, Expectation Maximization

\begin{abstract}
:
Direct radiometric comparison-based change detection methods have been widely used for detecting land cover change areas. However, the traditional methods are usually developed by using a single change index, which may cause omission or commission of land cover change. To address this challenge, we propose a change difference map fusion-based land cover change detection approach. First, operators of change vector analysis (CVA) and spectral gradient difference (SGD) are used for constructing change difference map, respectively. Second, image product weighted fusion method is introduced to construct a comprehensive change difference map. Finally, the expectation maximization algorithm and Bayesian rule with minimum error rate are applied to get the change/unchanged area. Experimental results of Landsat5 TM in 2010 and Landsat8 OLI in 2015 in two test areas are conducted. The experimental results show that accuracy of the proposed method is superior to the traditional change detection methods.
\end{abstract}

\section{INTRODUCTION}

Land cover change detection with remote sensed imagery is the process of identifying changed areas and types based on the images acquired at different times (Lu et al., 2004). With the development of image acquisition and image processing technologies, change detection has become an active research topic, which is widely used in many social benefit areas, such as land cover updating, urban expansion, land management and hazard assessment.

During the last twenty years, a large number of change detection algorithms and models have been developed and used. In general, change detection methods can be classified into direct radiometric comparison (DRC) and post-classification comparison (PCC) methods (Hussain et al., 2013). In DRC methods, a change difference map is constructed by using change index, which is further used to get the changed area by selecting an optimal threshold. PCC methods get both the changed areas and types by comparing two classification results based on the remote sensed images acquired from different dates. DRC methods are simpler and more straightforward than PCC. Besides, and DRC methods can avoid the accumulative error from image classification in the PCC methods. Due to these reasons, DRC methods have been applied widely in practice.

The selection of change index is the key step in the DRC methods. In current researches, the typical change indices include change vector analysis (CVA), spectral gradient difference (SGD), spectral angle difference (SAD), image difference (ID) and ratio difference (RD). These change indices often have their own advantages and drawbacks, there exist no single one can be universally applicable for all kinds of imagery, land cover types and geographic regions (Xing et al., 2017). In general, a single change index is often designed for a specific change detection task, which may cause omission or commission of land cover change. To address this problem, some researchers integrated the advantages and disadvantages of different change indices to construct a comprehensive change difference map for detecting the land cover changes more comprehensively and accurately. For example, $\mathrm{Ma}$ et al. constructed a change difference map by integrating the image difference map and ratio difference map (Ma et al., 2006). Mu et al. introduced the wavelet fusion method to combine the difference map obtained by ID index with that obtained by RD index (Mu et al., 2015). However, only the ID index and RD index were simply selected for constructing the fused change difference map, which still have some limitations for land cover change detection, especially in a complex and large area. To take advantage of more change indices, $\mathrm{Du}$ et al. proposed a novel change detection approach based on fusion of multiple spectral change difference images (Du et al., 2012). Feature and decision level fusion technologies were then used to combine the change difference maps generated by five different change indices.

In the current studies, the change indices for change detection can be classified into spectral value-based indices (SVI) and spectral shape-based indices (SSI). The SVI compare the spectral value of different times to identify the change area, these indices include CVA, ID, etc. The preferable effect can be achieved using SVI for the well-condition remote sensed images, however, the change results are be easily influenced by soil humidity, water turbidity or solar radiation conditions of the participant remote sensed images. The SSI compare the spectral shape of different times to identify the change area these indices include SGD, RD, etc. The pseudo changes can be reduced, which are caused by the spectral value changed while the spectral shape not changed. However, it is not sensitive for land cover types that possess lower spectral value. These two types of change indices have both advantages and disadvantages. In our previous researches, we have elaborated that SGD index and CVA index are the relative optimal method in SVI and SSI, respectively ( $\mathrm{Lu}$ et al., 2013). In this paper, we propose an image fusion approach-based change detection method. A comprehensive change difference map is firstly constructed by integrating CVA and SGD change indices for improving the change detection results. An EM algorithm-based change 
threshold selection method is then adopted for detecting the land cover change areas.

The paper proceeds as follows to achieve the above contribution. Section 2 describes the change detection method from the following three aspects: change magnitude computation, fusion difference map construction and change area detection. Section 3 presents two experiments to evaluate the effectiveness of the proposed method. Section 4 gives the conclusion and future works.

\section{METHODOLOGY}

\subsection{Change difference computing based on CVA and SGD}

In this paper, the CVA index and SGD index are selected for obtaining the change difference map. In CVA index, the spectral value of each band from different images are calculated to obtain the changes of all pixels, which is then forms the change vector (Chen et al., 2003). The change magnitude is calculated using Euclidean distance. In SGD index, the shape of the spectral curve is described by the gradient of different bands, which is further forms the spectral gradient vector (SGV) (Chen et al., 2013). Supposing there are $n$ bands in remote sensed image, $G_{C V A}$ and $G_{S G D}$ represent the change magnitude based on CVA and SGD indices, respectively. $V_{l i}$ and $V_{2 i}$ represent the spectral value of the $i$ bands in the $T 1$ time and $T 2$ time of images, respectively. $\lambda_{1 i}$ and $\lambda_{2 i}$ represent the wavelength of the $i$ bands in the $T 1$ time and T2 time of images, respectively. The formulas to compute the CVA and SGD indices are shown as the following formulas (3)-(5):

$$
\begin{gathered}
G_{C V A}=\sqrt{\sum_{i=1}^{n}\left(V_{2 i}-V_{1 i}\right)^{2}} \\
G_{S G D}=\sum_{i=1}^{n}\left|\frac{V_{2, i+1}-V_{2 i}}{\lambda_{2, i+1}-\lambda_{2 i}}-\frac{V_{1, i+1}-V_{1 i}}{\lambda_{1, i+1}-\lambda_{1 i}}\right|
\end{gathered}
$$

\subsection{Image product weighted fusion method-based change difference map fusion}

The change difference map based on CVA and SGD indices are integrated for getting the comprehensive difference map. In this paper, the algebraic weighted product transform fusion method was proposed to construct fusion image by improving the traditional image product method, in order to extract more richness and accurate change information. The adopted fusion formulas are shown as the following formulas (3)-(5):

$$
\begin{gathered}
G_{C M I F, i j}=f_{1 i j} G_{C V A, i j}+f_{2 i j} G_{S G D, i j} \\
f_{1 i j}=\frac{\frac{1}{k l} \sum_{i=1}^{k} \sum_{j=1}^{l}\left(G_{C V A, i j}-\overline{G_{C V A}}\right)\left(G_{S G D, i j}-\overline{G_{S G D}}\right)}{\sqrt{\frac{1}{k l} \sum_{i=1}^{k} \sum_{j=1}^{l}\left(G_{C V A, i j}-\overline{G_{C V A}}\right)^{2} \frac{1}{k l} \sum_{i=1}^{k} \sum_{j=1}^{l}\left(G_{S G D, i j}-\overline{G_{S G D}}\right)^{2}}} \\
f_{1 i j}+f_{2 i j}=1
\end{gathered}
$$

In the above formulas, $G_{C V A, i j}, G_{S G D, i j}$ and $G_{M I C D, i j}$ represent the change magnitude value of pixel $i j$ based on CVA, SGD and CMIF indices, respectively. $\overline{G_{C V A}}$ and $\overline{G_{S G D}}$ represent the average value of all pixels in the change difference map computing from CVA and SGD, respectively. $k$ and $l$ represent the number of rows and columns. $f_{l i j}$ and $f_{2 i j}$ represent the weight of $G_{C V A}$ and $G_{S G D}$ based on the correlation coefficient. A greater $G_{C M I F}$ indicates a larger change between the pixels in different times.

\subsection{Change area detection using EM algorithm and Bayesian rule}

On the basis of the comprehensive change difference map, suitable threshold should be selected for computing the change area. In this paper, the pixels in the change difference map are divided into the changed pixels $\omega_{c}$ and the unchanged pixels $\omega_{u}$. The theory of Bayesian rule with minimum error rate is used to compute the optimal change threshold (Bruzzone et al., 2000). Supposing that the prior probability and conditional probability density of the changed and unchanged pixels are represented as $p\left(\omega_{u}\right)$ and $p\left(\omega_{c}\right), p\left(x \mid \omega_{u}\right)$ and $p\left(x \mid \omega_{c}\right)$, and $p\left(\omega_{u}\right)+p\left(\omega_{c}\right)=1$. The formulas for computing the optimal change threshold is shown as follows.

$$
P\left(\omega_{u}\right) P\left(T \mid \omega_{u}\right)=P\left(\omega_{c}\right) P\left(T \mid \omega_{c}\right)
$$

Supposing that the conditional probability density function of changed and unchanged pixels follows Gaussian distribution, which is represented as formula (7).

$$
P\left(X \mid \omega_{i}\right)=\frac{1}{\sqrt{2 \pi \delta_{i}^{2}}} \exp \left\{-\frac{\left(x-m_{i}\right)^{2}}{2 \delta_{i}^{2}}\right\}
$$

In the above formula, $\omega_{i} \in\left(\omega_{u}, \omega_{c}\right)$, the mean value and standard deviation of $\omega_{i}$ are represented as $m_{i}$ and $\delta_{i}$. There have four unknown parameters in the solution of $p\left(X \mid \omega_{i}\right)$, including the mean value and standard deviation of changed pixels $\omega_{c}$ and unchanged pixels $\omega_{u}$, which are represented as $m_{u}, m_{c}, \delta_{u}, \delta_{c}$. This problem is typical for solving the missing parameters. In this paper, the expectation maximization (EM) algorithm is used for the solution of the above parameters. EM is a commonlyused algorithm for maximum likelihood estimation of the missing parameters, which compute the estimation of parameters only from observation data itself without any external data and prior knowledge. Iterative paradigm is adopted in EM algorithm for computing, the iterative formulas for the estimation of unchanged pixels $\omega_{u}$ are represented as (8)-(10).

$$
\begin{array}{r}
P^{t+1}\left(\omega_{u}\right)=\frac{\sum_{X(i, j) \in X_{d}} \frac{P^{t}\left(\omega_{u}\right) P^{t}\left(X(i, j) / \omega_{u}\right)}{P^{t}(X(i, j))}}{I^{*} J} \\
m_{u}{ }^{t+1}\left(\omega_{u}\right)=\frac{\sum_{X(i, j) \in X_{d}} \frac{P^{t}\left(\omega_{u}\right) P^{t}\left(X(i, j) / \omega_{u}\right) X(i, j)}{P^{t}(X(i, j))}}{I^{*} J} \\
\left(\delta_{u}^{2}\right)^{t+1}\left(\omega_{u}\right)=\frac{\sum_{X(i, j) \in X_{d}} \frac{P^{t}\left(\omega_{u}\right) P^{t}\left(X(i, j) / \omega_{u}\right)}{P^{t}(X(i, j))}\left[X(i, j)-m_{u}^{t}\right]^{2}}{\sum_{X(i, j) \in X_{d}} \frac{P^{t}\left(\omega_{u}\right) P^{t}\left(X(i, j) / \omega_{u}\right)}{P^{t}(X(i, j))}}
\end{array}
$$

In the above formulas, $t$ and $t+1$ represent the estimation value for $t$ and $t+1$ times of iteration. $I$ and $J$ are represent the number of rows and columns of difference map, $X(i, j)$ represents the pixel value of row $i$ and column $j$ of difference map. Before iteration, the initial value of $p\left(\omega_{i}\right), \omega_{i}$ and $\delta_{i}$ are obtained using K-means clustering algorithm. The iteration will be finished only the difference of $p\left(\omega_{i}\right), \omega_{i}$ and $\delta_{i}$ of two adjacent iterative calculation is less than a given threshold $\varepsilon\left(\varepsilon=10^{-8}\right)$. The parameters of the last iteration are used as the final results. The solution of the parameters of changed pixel $\omega_{c}$ is also following this process. The solution formulas for optimum threshold will be constructed by bring the obtained parameters into formula (6) and (7), which is represented as formula (11). $X(i, j) \in \omega_{u}$ only if $X(i, j) \leq T$. 
$\left(\delta_{u}^{2}-\delta_{c}^{2}\right) T^{2}+2\left(\mu_{u} \delta_{u}^{2}-\mu_{c} \delta_{c}^{2}\right) T+\mu_{c}^{2} \delta_{u}^{2}-\mu_{u}^{2} \delta_{c}^{2}-2 \delta_{u}^{2} \delta_{c}^{2} \ln \left[\frac{\delta_{u} P\left(\omega_{u}\right)}{\delta_{c} P\left(\omega_{c}\right)}\right]=0$

\section{EXPERIMENTS AND ANALYSIS}

\subsection{Study area and data}

In this paper, Landsat 5 TM of 2010 and Landsat 8 OLI of 2015 are selected as the data source for change detection. The selected images are acquired from the same season $(2010 / 06 / 22$ and 2015/06/12) with less cloud, which can reduce the influence from the solar elevation angle, seasonal difference for change detection. The pre-processing work include radiometric correction and geometric correction. First, ENVI 5.1 software is used for radiometric correction, the DN (Digital Number) is

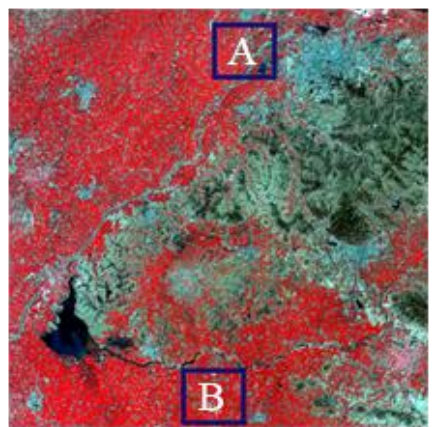

(a)

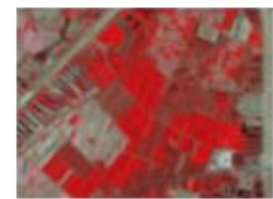

(b)

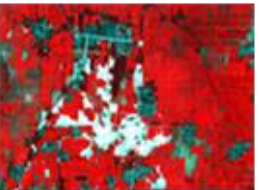

(d) converted into surface reflectance. Then, the geometric registration of two images is achieved within half a pixel.

The Midwest of Shandong province is selected as the study area, the Landsat number is P122R035, which are shown as Figure 1 (a). Two sub-area is selected for demonstration. Sub-area A is located in Qihe country Dezhou city, with $108 * 86$ pixels. Land cover changes in this area during 2010 to 2015 are mainly composed of water change. The Landsat images (432) of subarea A in 2010 and 2015 are shown as Figure 1 (b) and Figure 1 (c). Sub-area A is located in Wenshang country Jining city, with $318 * 278$ pixels. Land cover changes in this area during 2010 to 20156 are mainly composed of build-area change. The Landsat images (432) of sub-area B in 2010 and 2015 are shown as Figure $1(\mathrm{~d})$ and Figure 1 (e).

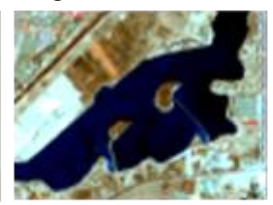

(c)

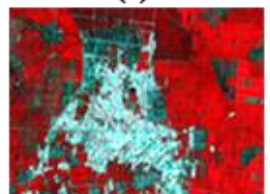

(e)

Figure 1. Location of the study areas and their composite images

(a) Location of the two-study area; (b) Sub-area A 2010; (c) Sub-area A 2015; (d) Sub-area B 2015; (e) Sub-area B 2015

\subsection{Accuracy assessment}

In order to evaluate the effectiveness of the proposed method, the accuracy of change detection based on CVA index, SGD index, multi-indices (Wei et al., 2010) and the proposed method are compared and analysed. The change difference maps are firstly constructed using these four change indices. The change difference maps of Sub-area A and B are shown as Figure 2 (a)(d) and Figure 4 (a)-(d), respectively. In these figures, brighter

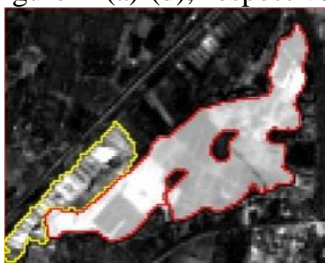

(a) CVA index

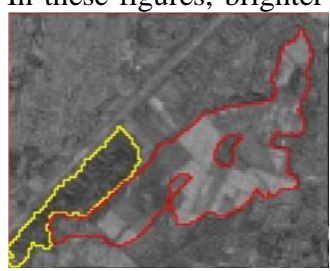

(b) SGD index

area indicates a larger change between the pixels in different times. Then, EM algorithm and Bayesian rule with minimum error rate are applied to select optimum threshold for getting the change/unchanged area. The thresholds of Sub-area A and B were $54,155,100,105.95$ and $71.56,82,27,39$, respectively. The change area of Sub-area A and B are shown as Figure 3 (a)(d) and Figure 5 (a)-(d), respectively. In the change area maps, black area represents the changed area, and white area represent unchanged area.

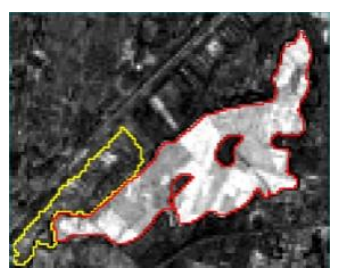

(c) multi-indices

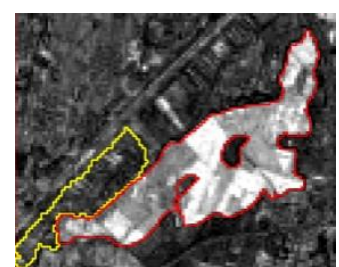

(d) the proposed indices

Figure 2. Change difference maps of sub-area A

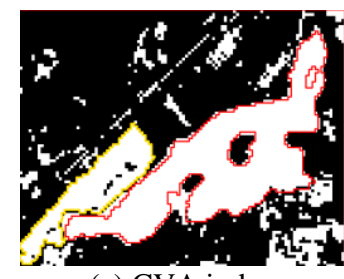

(a) CVA index

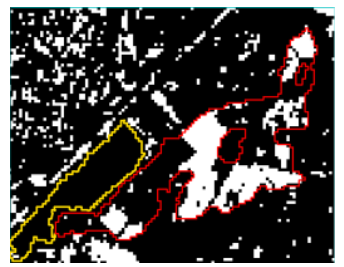

(b) SGD index

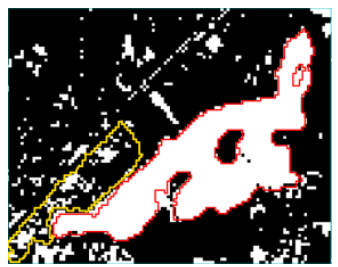

(c) multi-indices

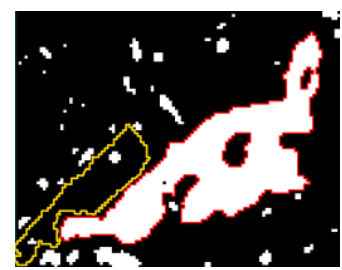

(d) the proposed indices

Figure 3. Change area maps of sub-area A 


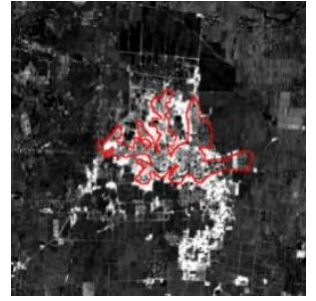

(a) CVA index

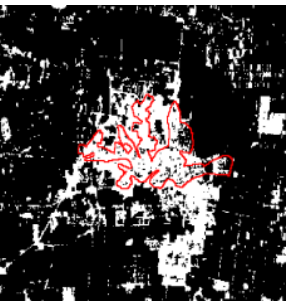

(a) CVA index

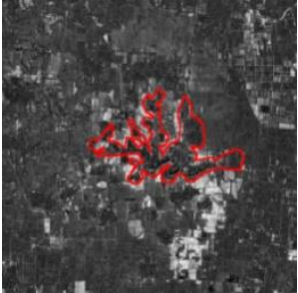

(b) SGD index

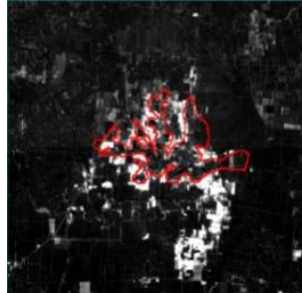

(c) multi-indices

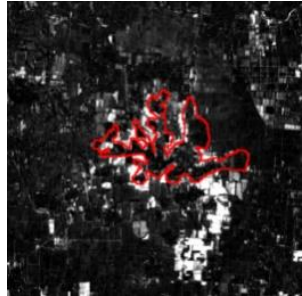

(d) the proposed indices

Figure 4. Change difference maps of sub-area B

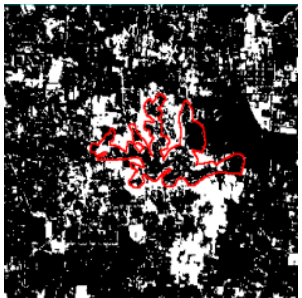

(b) SGD index

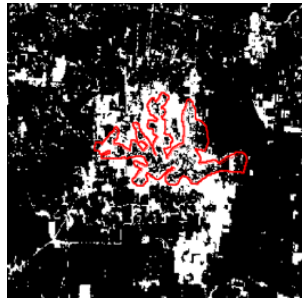

(c) multi-indices

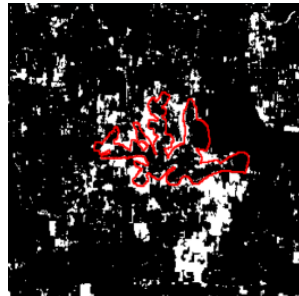

(d) the proposed indices

Figure 5. Change area maps of sub-area B

In order to evaluate the performance of the proposed method, samples were randomly taken for assessing the accuracy of the change area maps. The samples for sub-area A and B consisted of 102 changed pixels and 222 unchanged pixels, 259 changed pixels and 667 unchanged pixels, respectively. These samples were selected as the truthful data for computing the accuracy index of the above methods. The commission ratio, omission ration and overall accuracy were used for evaluation of the change detection results. The commission ration defines the percentage of false change caused by detection errors by using the ration of false detected changes to total detected changes. The omission ration defines the percentage of omission change caused by detection errors by using the ration of undetected changes to total true changes. Overall ratio describes the percentage of correct changed and unchanged detection pixels to the amount of testing samples. The higher overall accuracy and the lower commission ration and omission ration indicate the higher change detection accuracy. The accuracy index based on the above four methods for sub-area A and B are shown as Table 1 and 2, respectively.

Table 1 Accuracy of four change detection methods for sub-area A

\begin{tabular}{|c|c|c|c|c|c|c|c|c|}
\hline & \multicolumn{2}{|c|}{ CVA } & \multicolumn{2}{|c|}{ SGD } & \multicolumn{2}{|c|}{ multi-indices } & \multicolumn{2}{|c|}{ CMIF } \\
\hline & $\begin{array}{c}\text { Truthful } \\
\text { change }\end{array}$ & $\begin{array}{c}\text { Truthful } \\
\text { unchanged }\end{array}$ & $\begin{array}{l}\text { Truthful } \\
\text { changed }\end{array}$ & $\begin{array}{c}\text { Truthful } \\
\text { unchanged }\end{array}$ & $\begin{array}{l}\text { Truthful } \\
\text { changed }\end{array}$ & $\begin{array}{c}\text { Truthful } \\
\text { unchanged }\end{array}$ & $\begin{array}{l}\text { Truthful } \\
\text { changed }\end{array}$ & $\begin{array}{c}\text { Truthful } \\
\text { unchanged }\end{array}$ \\
\hline changes & 99 & 62 & 47 & 37 & 100 & 38 & 100 & $\overline{18}$ \\
\hline unchanged & 3 & 160 & 55 & 185 & 2 & 184 & 2 & 204 \\
\hline Total & 102 & 222 & 102 & 222 & 102 & 222 & 102 & 222 \\
\hline CR (\%) & \multicolumn{2}{|c|}{27.93} & \multicolumn{2}{|c|}{16.67} & \multicolumn{2}{|c|}{17.12} & \multicolumn{2}{|c|}{8.11} \\
\hline OR $(\%)$ & \multicolumn{2}{|c|}{2.94} & \multicolumn{2}{|c|}{53.92} & \multicolumn{2}{|c|}{1.96} & \multicolumn{2}{|c|}{1.96} \\
\hline $\mathrm{OA}(\%)$ & \multicolumn{2}{|c|}{79.94} & \multicolumn{2}{|c|}{71.60} & \multicolumn{2}{|c|}{87.65} & \multicolumn{2}{|c|}{93.83} \\
\hline
\end{tabular}

Table 2 Accuracy of four change detection methods for sub-area B

\begin{tabular}{|c|c|c|c|c|c|c|c|c|}
\hline & \multicolumn{2}{|c|}{ CVA } & \multicolumn{2}{|c|}{ SGD } & \multicolumn{2}{|c|}{ multi-indices } & \multicolumn{2}{|c|}{ CMIF } \\
\hline & $\begin{array}{l}\text { Truthful } \\
\text { change }\end{array}$ & $\begin{array}{c}\text { Truthful } \\
\text { unchanged }\end{array}$ & $\begin{array}{c}\text { Truthful } \\
\text { change }\end{array}$ & $\begin{array}{c}\text { Truthful } \\
\text { unchanged }\end{array}$ & $\begin{array}{l}\text { Truthful } \\
\text { change }\end{array}$ & $\begin{array}{c}\text { Truthful } \\
\text { unchanged }\end{array}$ & $\begin{array}{l}\text { Truthful } \\
\text { change }\end{array}$ & $\begin{array}{c}\text { Truthful } \\
\text { unchanged }\end{array}$ \\
\hline changes & 182 & 160 & 235 & 196 & 219 & 140 & 214 & 83 \\
\hline unchanged & 77 & 507 & 24 & 471 & 40 & 527 & 45 & 584 \\
\hline Total & 259 & 667 & 259 & 667 & 259 & 667 & 259 & 667 \\
\hline $\mathrm{CR}(\%)$ & \multicolumn{2}{|c|}{23.99} & \multicolumn{2}{|c|}{29.39} & \multicolumn{2}{|c|}{20.99} & \multicolumn{2}{|c|}{12.44} \\
\hline OR $(\%)$ & \multicolumn{2}{|c|}{29.73} & \multicolumn{2}{|c|}{9.27} & \multicolumn{2}{|c|}{15.44} & \multicolumn{2}{|c|}{17.37} \\
\hline $\mathrm{OA}(\%)$ & \multicolumn{2}{|c|}{74.41} & \multicolumn{2}{|c|}{76.24} & \multicolumn{2}{|c|}{80.56} & \multicolumn{2}{|c|}{86.18} \\
\hline
\end{tabular}

\subsection{Result analysis}

It can be seen from table 1 and 2, the overall accuracy of the proposed method is the highest, which could achieve $93.86 \%$ for sub-area A and $86.18 \%$ for sub-area B. There have too many pseudo changes in change area map that only using CVA method. As shown in Figure 3 (a) and Figure 5 (a), the yellow polygon area of Figure 3 (a) and red polygon area of Figure 5 (a) are mainly built-up areas, there have nearly no change during 2010 to 2015. However, influenced by solar altitude angle, illumination condition and other factors, the spectral value of images acquired in different times has changed greatly. There have many truthful changes that no be detected only using SGD 
method. As shown in the red polygon area of Figure 3 (b), the water changes were not fully detected. This is mainly because that water type is a land cover type that have lower spectral value, which is difficult to detect truthful changes for these land cover types only using the spectral shape-based change detection methods. In the method of multi-indices (Wei et al., 2010), weighted product image fusion method was used for change difference map fusion of difference index and ratio index. The overall accuracy of this method is higher than CVA method and SGD method. The advantages of spectral valuebased index and spectral shape-based index are synthesized in the proposed method. In addition, all bands of Landsat image were used for change detection. Therefore, the accuracy of the proposed method is higher than the method based on multiindices (Wei et al., 2010). From the comparison of change detection results, accuracy and analysis, the comprehensive and accurate change area could be obtained using the proposed method, and the change detection accuracy could be also effectively improved.

\section{CONCLUSION}

In this paper, we proposed a novel land cover change detection method based on change map fusion. CVA and SGD indices were firstly used for constructing change magnitude image, respectively. Then, image product weighted fusion method was introduced to construct a comprehensive change difference map. At last, the expectation maximization algorithm and Bayesian rule with minimum error rate was applied to get the change/unchanged area. Experimental results of Landsat5 TM in 2010 and Landsat8 OLI in 2015 in two test areas showed that accuracy of the proposed method is superior to the traditional change detection methods.

The unified optimum threshold was selected for all land cover types to get change area. In fact, different land cover type should have different change threshold. In future, self-adaption change threshold selection method should be developed according to the characteristic of different land cover types.

\section{ACKNOWLEDGEMENTS}

This work has been funded by the National Science Foundation of China (Project \#41801308, \#41701443) and Doctoral Research Fund of Shandong Jianzhu University (XNBS1804).

\section{REFERENCES}

Bruzzone, L., Prieto, D. F., 2000. Automatic analysis of the difference image for unsupervised change detection. Geoscience and Remote Sensing, IEEE Transactions on Geoscience \& Remote Sensing, 38(3), 1171-1182.

Chen, J., Gong, P., He, C., Pu, R., Shi, P., 2003. Land-use/landcover change detection using improved change-vector analysis. Photogrammetric Engineering \& Remote Sensing, 69(1), 369379.

Chen, J., Lu, M., Chen, X., Chen, J., Chen, L., 2013. A spectral gradient difference based approach for land cover change detection. ISPRS Journal of Photogrammetry and Remote Sensing, 85(2), 1-12.

Du P., Liu S., Gamba P., Tan, K., Xia, J., 2012. Fusion of Difference Images for Change Detection Over Urban Areas. IEEE Journal of Selected Topics in Applied Earth Observations \& Remote Sensing, 5(4), 1076-1086.
Hussain, M., Chen, D., Cheng, A., Wei, H., Stanley, D., 2013. Change detection from remotely sensed images: From pixelbased to object-based approaches. ISPRS Journal of Photogrammetry and Remote Sensing, 80, 91-106.

Lu, D., Mausel, P., Brondizio, E., Moran, E., 2004. Change detection techniques. International Journal of Remote Sensing $25,2365-2401$.

Lu, M., Mei, Y., Chen, L., 2013. Land Cover Change Detection Based on Value and Shape Optimized Combination. Geomatics and Information Science of Wuhan University, 38(6), 669-673.

Ma, G., Li, P., Qin, Q., 2006. Based on Fusion and GGM Change Detection Approach of Remote Sensing Images. Journal of Remote Sensing, 10(6), 847-853.

Mu, C., Huo, L., Liu, Y., Liu, R., Jiao, L., 2015. Change Detection for Remote Sensing Images Based on Wavelet Fusion and PCA-Kernel Fuzzy Clustering. Acta Electronica Sinica, 43(7), 1375-1381.

Wei L., Zhong Y., Zhang L., 2010. Adaptive change method of remote sensing image fusion. Journal of Remote Sensing, 14(6), 1196-1211.

Xing H., Chen J., Wu H., Zhang, J., Li, S., Liu, B., 2017. A service relation model for web-based land cover change detection. International Journal of Photogrammetry \& Remote Sensing, 132:20-32. 